\title{
Long-term follow-up of children with congenital chylothorax
}

\section{To the Editors:}

Congenital chylothorax, defined as the accumulation of lymphatic fluid in the pleural cavity, is a rare neonatal disorder. Nevertheless, congenital chylothorax represents the most common cause of pleural effusion in fetuses and newborns [1]. Due to the rarity of this disorder only a few case series are reported in the literature and current treatment recommendations are based on individual case reports or case series only [2-5]. The diagnosis of chylothorax is considered when pleural fluid triglyceride levels are $>1.1 \mathrm{mmol} \cdot \mathrm{L}^{-1}$ and total cell counts are $>1,000$ cells per $\mathrm{mL}$ with $>80 \%$ lymphocytes. Without oral fat intake chylomicrons, which are often an indication for the diagnosis of chylothorax, are absent in the pleural fluid and distinction between chylous and non-chylous effusion might be difficult [6]. Long-term follow-up of children with congenital chylothorax, however, was documented in only four studies [7-10] and none included pulmonary function testing. We describe the clinical course and long-term outcome of infants with congenital chylothorax including neurodevelopmental follow-up and pulmonary function testing.

In this study the data of all children who were documented in the local database of the Division of Neonatology (Paediatric Dept, Medical University of Graz, Graz, Austria) with the diagnosis of congenital chylothorax between January 1, 1995 and December 31, 2009 were retrospectively analysed. Congenital chylothorax was diagnosed by thoracentesis and biochemical analysis of aspirated fluid (lymphocytes $>80 \%$ of total cell numbers or the presence of chylomicrons in newborns with previous enteral nutrition). Neonates who suffered from postoperative or iatrogenic chylothorax were excluded from analysis. Clinical, radiological and laboratory data were analysed including sex, gestational age, Apgar score, localisation of the pleural effusion, associated malformations, chromosomal abnormalities, duration of the effusion, duration of mechanical ventilation, occurrence of nosocomial infections, prenatal and postnatal treatment and duration of hospitalisation.

From April to August 2010 a prospective follow-up investigation was performed including a survey by a structured questionnaire (the childs development including data from our outpatient clinic of neurodevelopmental follow-up, medical problems, hospitalisations, medications and respiratory tract problems), a subsequent physical examination and pulmonary function testing. Pulmonary function testing included spirometry and body plethysmography (MasterLab, Jaeger, Würzburg, Germany) and was performed according to European Respiratory Society (ERS) and American Thoracic Society (ATS) standards [11]. The study was approved by the local Ethics Committee (Nr. 21-182 ex 09/10).

Eight children with the diagnosis of congenital chylothorax were documented in the database and two had to be excluded (one with an iatrogenic cause of chylothorax and another one without biochemical analysis of the pleural fluid) thus, six children were considered to have congenital chylothorax resulting in an incidence of 4.6:100,000 deliveries in our region. The neonatal data are summarised in table 1 . Three cases underwent treatment with octreotide for 27, 29, and 31 days, respectively, and one required surgical intervention at day 54 . Associated anomalies included congenital heart disease (atrial septal defect, pulmonary artery stenosis) in association with Noonan syndrome and interstitial lung disease (pulmonary eosinophilic vasculitis). All infants suffered from hypoalbuminaemia, electrolyte imbalances, clotting dysfunction, lymphopenia, anaemia and immune deficiency due to chronic loss of immunoglobulins.

Long-term follow-up at a mean (range) age of 7 (3.5-12) yrs revealed no recurrence of chylothorax. All children except for the case diagnosed with Noonan syndrome attained a normal height and weight according to their age (table 2). Four children were re-hospitalised mainly due to infectious diseases and three had recurrent respiratory tract infections (table 2). One child diagnosed with asthma required regular medication with fluticasone propionate. One child developed high-functioning autism with neurodevelopmental delay and muscular hypotonia, another child aged 7 yrs had a delay in speech development because both parents were deaf, thus, five out of six children were classified as having developed normally.

Pulmonary function testing in five children revealed slightly abnormal results in three of them (borderline restrictive lung disease and a mild increase in airway resistance in case one with interstitial lung disease; mild curvilinearity of the maximal flow-volume curve in the otherwise healthy case two; and mild airway obstruction with a significant bronchodilator response in case five). Spirometry in case five was normal but spirometry was not possible in case six. Details are provided in table 2.

We report for the first time pulmonary function testing results revealing only slightly abnormal findings that might be partially explained by co-morbidities (table 2). Due to the complicated neonatal course of disease, including the long-term need for ventilatory support and supplemental oxygen, pulmonary damage might be suspected. But in all cases pulmonary function testing results overall demonstrate normal findings. This is an important issue for counselling parents at the neonatal ward during the first weeks of life when congenital chylothorax is associated with many complications. Intercurrent upper and lower respiratory tract infections and two cases of pneumonia did not appear to have influenced results of pulmonary function testing. Our results are somewhat limited by the small number of cases associated with the rarity of congenital chylothorax. Neurodevelopmental outcome seems primarily to depend on associated anomalies and/or prematurity and was in five of the six cases normal and age-appropriate. ERGAZ et al. [8] who followed seven out of 11 cases at a median (range) age of 30 (14-96) months, diagnosed five of them as having developed normally. The remaining two children included one child 


\begin{tabular}{lc}
\hline TABLE 1 & $\begin{array}{l}\text { Perinatal data of the six cases with diagnosis of } \\
\text { congenital chylothorax }\end{array}$ \\
\hline Gestational age weeks & $35.6 \pm 2.4(33.1-39.6)$ \\
Birth weight g & $2603 \pm 680(1740-3788)$ \\
Gestational age at diagnosis weeks & $33 \pm 3.6(30-39)$ \\
Bilateral pleural effusions & $6 / 6$ \\
Intrauterine thoracocentesis & $3 / 6$ \\
Hydrops fetalis & $2 / 6$ \\
Amniocentesis and genetic testing & $3 / 6$ \\
Associated anomalies & $3 / 6$ \\
Apgar sore at $\mathbf{5}$ min & $7.3 \pm 1.6(5-9)$ \\
Duration of mechanical ventilation days & $34(9-53)$ \\
Duration of supplemental oxygen days & $29(6-66)$ \\
Inotropic support for arterial hypotension & $5 / 6$ \\
Total parenteral nutrition days & $29(10-67)$ \\
Pneumothorax & $4 / 6$ \\
Late-onset sepsis & $3 / 6$ \\
Age at discharge days & $66(37-104)$ \\
\hline & \\
Data are presented as mean+ SD (range), number/total & or median (range), \\
unless otherwise stated. &
\end{tabular}

suffering from congenital pulmonary lymphangiectasis who required ventilatory support via tracheostomy and had severe motor and cognitive impairment including deafness and suspected cortical blindness and another child diagnosed with Klinefelter syndrome who attended a small special education class at a regular school. Thus, results confirm that neurodevelopmental outcome is mainly dependent on associated anomalies or a history of extreme prematurity.

From a series of 32 children nearly half of them were diagnosed as having asthma at a median follow-up of 7 yrs including four prematurely born infants (32-34 weeks) with recurrent respiratory tract infections [7]. In the mid-term follow-up, the authors did not observe any recurrence of congenital chylothorax. In a study by MUSSAT et al. [9] three out of 11 children had chest radiograph abnormalities at long-term follow up. According to our pulmonary function testing results radiograph pathologies do not seem to predict functional residuals of congenital chylothorax. Other studies also reported on full recovery except for those cases with associated anomalies $[8,10]$.

In conclusion, the predominant complicated neonatal course of the disease resulted in an overall age-appropriate neurodevelopmental outcome in our case series, without major pulmonary impairments demonstrated by pulmonary function testing.

\section{Bernhard Resch*,\#, Melanie Halmer*, Wilhelm D. Müller ${ }^{\#}$ and Ernst Eber}

* Research Unit for Neonatal Infectious Diseases and Epidemiology, Medical University of Graz, "Division of Neonatology, Dept of Pediatrics, Medical University of Graz, and "Respiratory and Allergic Disease Division, Dept of Pediatrics, Medical University of Graz, Graz, Austria.

Correspondence: B. Resch, Division of Neonatology, Paediatric Dept, Medical University of Graz, Auenbruggerplatz 34/2, A-8036 Graz, Austria. E-mail: bernhard.resch@medunigraz.at

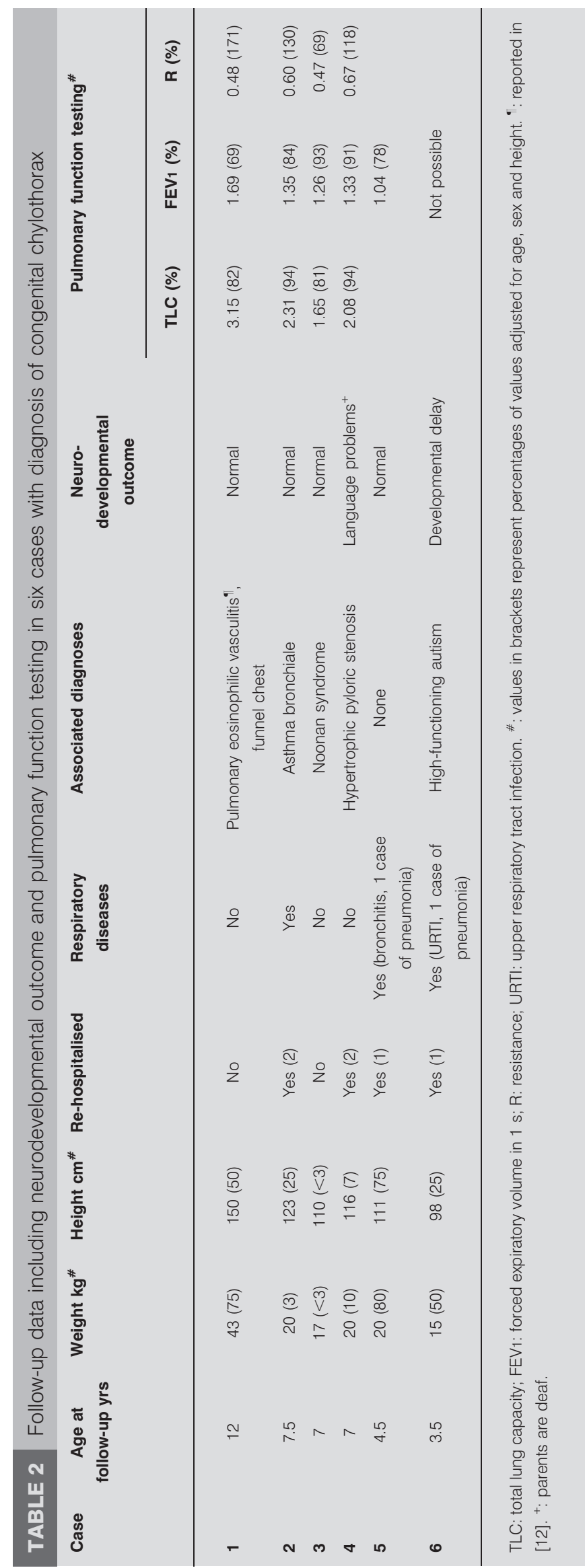

VOLUME 40 NUMBER 4 
Statement of Interest: None declared.

\section{REFERENCES}

1 Rocha G, Fernandes P, Rocha P, et al. Pleural effusions in the neonate. Acta Paediatr 2006; 95: 791-798.

2 Mitanchez D, Walter-Nicolet E, Salomon R, et al. Congenital chylothorax: what is the best strategy? Arch Dis Child Fetal Neonatal Ed 2006; 91: F153-F154.

3 Fernández Alvarez JR, Kalache KD, Graǔel EL. Management of spontaneous congenital chylothorax: oral medium-chain triglycerides versus total parenteral nutrition. Am J Perinatol 1999; 16: 415-420.

4 Nygaard U, Sundberg K, Nielsen HS, et al. New treatment of early fetal chylothorax. Obstet Gynecol 2007; 109: 1088-1092.

5 Rasiah SV, Oei J, Lui K. Octreotide in the treatment of congenital chylothorax. J Paediatr Child Health 2004; 40: 585-588.

6 Das A, Shah PS. Octreotide for the treatment of chylothorax in neonates. Cochrane Database Syst Rev 2010; 9: CD006388.
7 Caserío S, Gallego C, Martin P, et al. Congenital chylothorax: from foetal life to adolescence. Acta Paediatr 2010; 99: 1571-1577.

8 Ergaz Z, Bar-Oz B, Yatsiv I, et al. Congenital chylothorax: clinical course and prognostic significance. Pediatr Pulmonol 2009; 44: 806-811.

9 Mussat P, Dommergues M, Parat S, et al. Congenital chylothorax with hydrops: postnatal care and outcome following antenatal diagnosis. Acta Paediatr 1995; 84: 749-755.

10 Randolph JG, Gross RE. Congenital chylothorax. AMA Arch Surg 1957; 74: 405-419.

11 Frey U, Stocks J, Coates A, et al. Specifications for equipment used for infant pulmonary function testing. ERS/ATS Task Force on Standards for Infant Respiratory Function Testing. European Respiratory Society/American Thoracic Society. Eur Respir J 2000; 16: 731-740.

12 Resch B, Popper HH, Urlesberger B, et al. Pulmonary eosinophilic vasculitis in a neonate with congenital chylothorax. Pediatr Pulmonol 2002; 33: 501-504.

DOI: $10.1183 / 09031936.00016812$ 